\title{
Caracterización de la mortalidad por hidatidosis humana. Chile, 2000-2010
}

\author{
Paulina Martínez
}

\section{Characterization of human hydatidosis mortality. Chile, 2000-2010}

Background: Hydatidosis as a worldwide zoonosis with a high socio-economic impact in Chile and other countries for which it is a notifiable human disease in our country. Objective: To characterize death rates by hydatidosis and whether there are differences according to the region of residence. Material: A descriptive analysis using Data System Deaths (2000-2010) was performed. Results: The total number of deaths was 293 (56\% male). The mortality rate changed from 0.16 in 2000 to 0.11 in 2010 per 100.000 inhabitants. The main diagnosis corresponded to B67.9 (Echinococcosis, other and unspecified). Most fatal cases had a basic level of education and the Araucanía Region had the highest mortality rate. There was a loss of life of 5022.04 years by the premature death of 293 people, with a ratio of 0.31 PYLL years lost per 1000 inhabitants. In the Araucanía Region this ratio increased to 1.03 PYLL years lost per 1000 inhabitants. Conclusions: The mortality rate tends to decrease, however this trend might mask regional differences. It should be noted that deaths caused by hydatidosis are preventable deaths and therefore unjust. Finally, the mortality analysis is important to better understand the burden of the disease and evaluate the effectiveness of public health interventions.

Key words: Hydatidosis; human hydatidosis; deaths.

Palabras clave: Hidatidosis; hidatidosis humana; defunciones.

\section{Introducción}

\section{$\mathrm{L}$} a hidatidosis (o equinococosis) es una enfermedad producida por formas larvarias del género Echinococcus. Es reconocida como una zoonosis que en humanos genera alto impacto socio-económico. Por este motivo, el Ministerio de Salud de Chile (MINSAL) incorporó esta enfermedad al sistema de notificación obligatoria.

\section{Características generales}

La equinococosis humana está asociada a la ganadería de tipo extensiva, deficiencias en manejos sanitarios, bajos niveles socio-económicos y ausencia de educación sanitaria, incluyendo sistemas de matanza clandestina y condiciones inadecuadas de mataderos ${ }^{1}$.

Esta enfermedad zoonótica es causada por formas larvarias de varios géneros del parásito de la Clase Cestoda, Orden Cyclophyllidea, Familia Taeniidae, Género Echinococcus. Existen diferentes especies de Echinococcus, pero sólo cuatro-E. granulosus, E. multilocularis, E. oligarthrus y E. vogeli- son reconocidas como taxonómicamente relevantes y sólo las primeras dos son patogénicas para el humano ${ }^{1}$.

El ciclo de vida del parásito incluye dos clases de hospederos: definitivo o carnívoro (especialmente el perro), donde los parásitos desarrollan en el intestino la fase adul- ta o estrobilar, y el intermediario herbívoro u omnívoro (ovino, caprino, bovino o porcino) donde se desarrollan las formas larvarias o metacéstode, en los tejidos en forma de quiste ("quiste hidatídico") especialmente en el hígado y pulmón. El hombre se sitúa como hospedero accidental en el grupo de hospederos intermediarios ${ }^{2}$.

Los perros eliminan huevos del parásito en sus excrementos, los que se diseminan sobre su pelaje y contaminan el medioambiente. Los humanos ingieren estos huevos al acariciar sus perros y llevarse las manos a la boca o al trabajar en jardines y campos o por la ingestión de verduras o aguas contaminadas con materia fecal canina ${ }^{3}$.

En el intestino delgado del ser humano se liberan las larvas, penetran la pared, llegando mayoritariamente al hígado, donde forman quistes. También pueden formarse quistes en otros sitios como pulmones, huesos, cerebro, riñón, bazo y otros tejidos. Los quistes presionan los órganos donde se desarrollan y al expandirse provocan atrofia y posteriormente necrosis por presión en los tejidos circundantes. Por otra parte, una de las complicaciones más frecuentes es la rotura del quiste, lo que puede desencadenar una reacción anafiláctica y conduce a la siembra secundaria y formación de nuevos quistes ${ }^{4}$.

Esta zoonosis es considerada una enfermedad crónica, que afecta a personas de todas las edades pero, particularmente edades productivas, repercutiendo fuertemente sobre el individuo afectado, su entorno familiar y sobre la
Universidad Diego Portales, Santiago, Chile.

El estudio se realizó sin aporte económico o becas. No existe relación financiera o personal con personas u organizaciones que pudiesen dar lugar a conflicto de interés con este artículo.

Recibido: 28 de enero de 2013 Aceptado: 12 de diciembre de 2013

Correspondencia a: Paulina Martínez Gallegos paulinamartinezg@yahoo.com 
economía nacional, incluyendo los consiguientes costos por pérdidas laborales, consultas médicas y prestaciones anexas, cirugía y recuperación, licencias médicas, invalidez, gastos de hospitalización y medicamentos ${ }^{5-7}$. El contagio ocurre más fácilmente en los primeros años de vida dado que los hábitos de los menores de edad favorecen la ingestión de huevos desde las heces de los hospederos definitivos.

El diagnóstico se basa en datos epidemiológicos, clínicos, radiológicos y de laboratorio ${ }^{6}$. Se ha determinado que la ecografía es una técnica muy importante para el diagnóstico y seguimiento post tratamiento. La microscopia del líquido hidatídico es útil para confirmar la infección y viabilidad quística. También se describe que la resonancia magnética sería útil para determinar la viabilidad de los quistes y reconocer su estadio ${ }^{7}$. Mientras, la reacción de polimerasa en cadena (RPC) permitiría entregar un diagnóstico definitivo. Ninguna técnica por si sola otorga un diagnóstico infalible por lo cual es necesaria la complementariedad de técnicas diagnósticas ${ }^{6}$.

Históricamente, esta enfermedad ha sido considerada de resolución quirúrgica (mediante cirugía abierta o ecoasistida), donde se elimina el (los) quiste(s), y se procede a la corrección de sus efectos en el órgano afectado y el tratamiento de las posibles complicaciones ${ }^{7}$.

El Ministerio de Salud de Chile (MINSAL) incorporó esta enfermedad al sistema de notificación y según Acuña y cols., es obligatoria desde $1951^{8}$. Actualmente, en nuestro país es una enfermedad de notificación obligatoria (ENO) que se ratifica de acuerdo al Decreto Supremo No712, año 2000, cuyo objetivo es caracterizar epidemiológicamente los casos, forma de presentación y fuentes de infección, de manera de interrumpir la transmisión y desarrollar medidas de prevención y control en humanos. El sistema ENO establece la modalidad de notificar diariamente la sospecha de hidatidosis a la Autoridad Sanitaria Regional ${ }^{9,10}$.

\section{Situación epidemiológica}

La hidatidosis se encuentra ampliamente distribuida en el mundo, alcanzando altos niveles de incidencia en países como Argentina, Uruguay, Chile, Australia, Nueva Zelandia y en Europa, fundamentalmente en Grecia, Italia, Portugal y España ${ }^{6}$. Esta enfermedad afecta principalmente zonas o áreas rurales, o localidades con características rurales en los que generalmente el acceso a los servicios de salud por parte de la población es menor. Por lo tanto, sin duda la pobreza es decisiva en esta enfermedad ${ }^{11}$. En países endémicos como Perú, Brasil, Uruguay, Argentina y Chile no sólo se asocia a zonas rurales dedicadas a la ganadería de pastoreo - siendo el hospedero intermediario animal de mayor importancia epidemiológica el ovino- ${ }^{11}$ sino también a perfiles geográficos dados los diferentes nichos ecológicos y culturales ${ }^{12}$.
La complejidad de esta enfermedad está determinada por múltiples factores que participan en la exposición al agente y posterior desarrollo. Existen determinantes estructurales como el nivel educacional, nivel socioeconómico, patrones culturales, antropológicos y prácticas de producción agrícolas, que van a impactar en los patrones epidemiológicos y de distribución de la enfermedad.

En Chile, según el sistema de notificación ENO, los casos de hidatidosis humana han variado desde 297 en el año 2001 (52,2\% mujeres) a 267 en 2009 (52,4\% hombres), con un promedio anual de 311 casos, y tasas de notificación que se han desplazado desde 1,9 casos por 100 mil habts., en 2001 a 1,6 casos por 100 mil habts., en 2009, evidenciando una tendencia al decrecimiento. Respecto a la distribución geográfica, las mayores tasas de incidencia se registran en Aysén con 28,9 casos por 100 mil habts., en $2009^{13}$.

De acuerdo a los datos de los egresos hospitalarios, se registra una variación desde 1.150 egresos en 2001 (53,2\% hombres) a 498 en 2008 (52,4\% hombres), con un promedio anual cercano a 1.009 egresos. El mayor número de egresos se registró en 2003 (n: 1.224). La tasa de egresos hospitalarios se ha desplazado desde 7,4 a 3 egresos por 100 mil habts., con tendencia al decrecimiento. Según distribución geográfica, la mayor tasa de egresos hospitalarios es en Aysén con 55 egresos por 100 mil habts., en $2008^{13}$.

Las muertes clasificadas según CIE10 (códigos B67.0 a B67.9) han variado de 31 en 2001 (61,3\% hombres) a 15 en 2009 (53,3\% hombres), con un promedio anual cercano a 27,8 defunciones y una tasa país en el año 2009 de 0,1 muertes por 100 mil habts. La tasa de mortalidad país evidencia una tendencia al decrecimiento; sin embargo, este indicador esconde las realidades regionales. De acuerdo a las tasas de mortalidad regional, la mayor es en La Araucanía con 6,7 defunciones por 100 mil habts., lo que podría ser explicado por las diferencias en la calidad y acceso a las prestaciones que se entregan en los servicios de salud ${ }^{13}$. A modo de ejemplo, en Arica y Parinacota hay 15 médicos por cada 100 mil habts., comparado con La Araucanía con solamente uno ${ }^{5}$ por 100 mil habts.

Si bien la enfermedad afecta a todas las edades, es especialmente importante en edades productivas, repercutiendo fuertemente sobre el individuo afectado, su entorno familiar y sobre la economía nacional, tal como fue comunicado por esta autora ${ }^{13}$, donde la mayoría de los casos notificados se encuentra entre los 30 y 59 años de edad, con una edad mediana de 38 años, 39 años y 68 años, para los casos notificados, egresos hospitalarios y defunciones, respectivamente ${ }^{13}$.

Según la Organización Panamericana de la Salud (OPS), existen en el mundo enfermedades infecciosas, en su mayoría zoonosis, que representan una gran carga a los afectados, social y económica, las cuales han sido 
llamadas las "Enfermedades desatendidas" porque no son consideradas problemas de salud pública. Estas enfermedades generalmente no dan emergencias epidemiológicas destacadas por lo cual no llaman la atención de los medios de comunicación y entre los diferentes sectores ${ }^{14}$. La ganancia en salud, es decir, el efecto logrado al recibir una atención adecuada o efectiva, puede ser medida directa o indirectamente, siendo esta última la cuantificación de las pérdidas -ya sea discapacidad o muerte-, que se evitarían por una atención de salud oportuna y efectiva. Estos mismos autores, mencionan que el concepto de "muerte evitable" se discute desde hace décadas, y se refiere básicamente al hecho que la "atención médica efectiva y oportuna potencialmente evita muertes prematuras que no deben ocurrir", por lo tanto, también se refiere a la forma de distribución y acceso a la atención de salud ${ }^{15}$.

Las muertes producidas por hidatidosis son evitables, dado que existen formas para su control, diagnóstico y tratamiento. Y de acuerdo a la definición de la OPS y la Organización Mundial de la Salud (OMS), estas muertes expresarían inequidad debido a que son desigualdades "innecesarias, evitables e injustas"16.

\section{Objetivos}

Efectuar una caracterización general de la enfermedad humana en Chile y analizar la evolución de las defunciones durante el período 2000-2010, tratando de responder a la pregunta si existen diferencias en las defunciones según la región de residencia.

\section{Métodos}

\section{Diseño del estudio}

Estudio descriptivo. Se emplearon los datos secundarios provenientes del Sistema de Registro de Defunciones del Registro Civil/MINSAL/INE, entre los años 2000 y 2010. Se distinguieron según la Clasificación Internacional de Enfermedades (CIE10) los códigos B67.0 (Infección del hígado debida a E. granulosus); B67.1 (Infección del pulmón debida a E. granulosus); B67.2 (Infección de hueso debida a E. granulosus); B67.3 (Infección de otro órgano y de sitios múltiples por E. granulosus); B67.4 (Infección debida a E. granulosus, sin otra especificación); B67.5 (Infección del hígado debida a Echinococcus multilocularis); B67.6 (Infección de otros órganos y de sitios múltiples por E. multilocularis); B67.7 (Infección debida a E. multilocularis, sin otra especificación); B67.8 (Equinococosis del hígado, no especificada); B67.9 (Equinococosis, otra y la no especificada $)^{17}$.

\section{Diseño de análisis}

Se realizaron análisis uni y bivariado, describiendo las características de las personas como sexo, edad, diagnóstico, lugar de residencia, nivel de educación, ocupación y estado civil. También, se calcularon las tasas por año y región, utilizando la población $\mathrm{INE}^{18}$ a mitad de período, evaluando la variación temporal y geográfica.

A la vez, se realizó una estimación de las muertes evitables mediante los años de vida potencialmente perdidos (AVPP) y la razón de AVPP, empleando como límite arbitrario la esperanza de vida al nacer en el país y la población INE. Se compararon las muertes evitables según región de residencia, a través de los AVPP y la razón de AVPP.

Se emplearon los programas Excel, SPSS 10.0 y Stata 11.0.

\section{Resultados}

\section{Análisis uni y bivariado}

Entre los años 2000 y 2010 se registraron 293 defunciones por hidatidosis, las que se concentraron en $56 \%$ en hombres (n: 164) y 44\% en mujeres (n: 129). El número de defunciones ha variado, 25 en el año 2000 (52\% hombres) a 18 en el 2010 (55,6\% hombres), registrándose en 2007 el mayor número de defunciones (n: $35 ; 57 \%$ hombres). La Tabla 1 muestra el detalle de las defunciones según sexo.

La tasa de mortalidad a nivel país ha variado desde 0,16 muertes por 100 mil habts., en el año 2000 a 0,11 defunciones por 100 mil habts., en el 2010, registrándose en los años 2002 y 2007 la tasa máxima (0,21 muertes por 100 mil habts). Mientras en hombres se ha desplazado desde 0,17 a 0,12 muertes por 100 mil hombres, alcanzando el máximo en el año 2002 (0,28 defunciones por 100 mil hombres). Adicionalmente, en mujeres la tasa de mortalidad ha cambiado desde 0,15 a 0,09 fallecimientos por 100 mil mujeres, con registro de tasa máxima el 2006

Tabla 1. Número de defunciones y tasas* de mortalidad por hidatidosis. Chile, 2000-2010

\begin{tabular}{|ccccccc|}
\hline Año & $\begin{array}{c}\mathbf{n} \text { defunciones } \\
\text { País }\end{array}$ & $\begin{array}{c}\text { Tasa } \\
\text { país }\end{array}$ & $\begin{array}{c}\text { n defunciones } \\
\text { Hombre }\end{array}$ & $\begin{array}{c}\text { Tasa } \\
\text { hombre }\end{array}$ & $\begin{array}{c}\text { n defunciones } \\
\text { Mujer }\end{array}$ & $\begin{array}{c}\text { Tasa } \\
\text { mujer }\end{array}$ \\
\hline 2000 & 25 & 0,16 & 13 & 0,17 & 12 & 0,15 \\
2001 & 31 & 0,20 & 19 & 0,25 & 12 & 0,15 \\
2002 & 33 & 0,21 & 22 & 0,28 & 11 & 0,14 \\
2003 & 32 & 0,20 & 17 & 0,22 & 15 & 0,19 \\
2004 & 27 & 0,17 & 16 & 0,20 & 11 & 0,14 \\
2005 & 25 & 0,15 & 13 & 0,16 & 12 & 0,15 \\
2006 & 30 & 0,18 & 12 & 0,15 & 18 & 0,22 \\
2007 & 35 & 0,21 & 20 & 0,24 & 15 & 0,18 \\
\hline 2008 & 22 & 0,13 & 14 & 0,17 & 8 & 0,09 \\
2009 & 15 & 0,09 & 8 & 0,10 & 7 & 0,08 \\
\hline 2010 & 18 & 0,11 & 10 & 0,12 & 8 & 0,09 \\
\hline Total & 293 & & 164 & & 129 & \\
\hline * Tasas por 100 mil habts. & & & & & \\
\hline
\end{tabular}




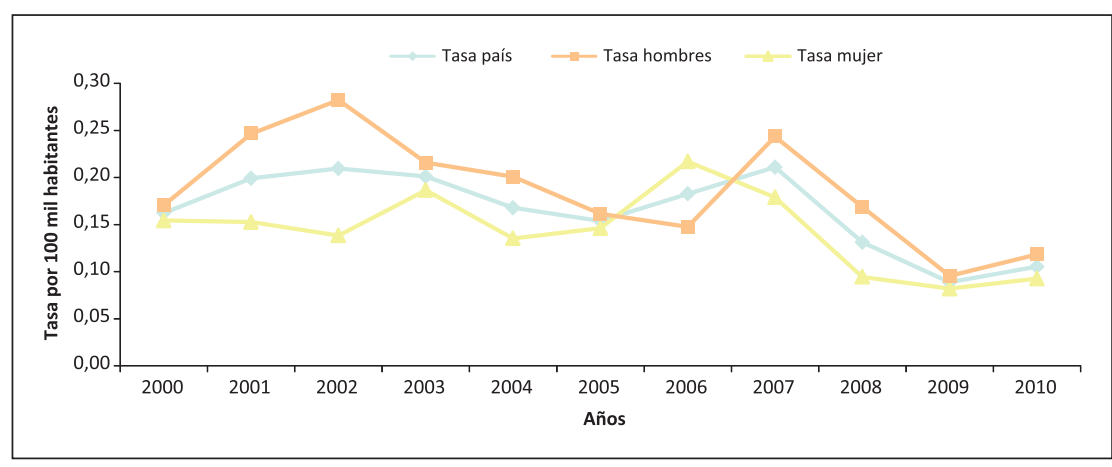

Figura 1. Tasa de defunciones por hidatidosis según sexo. Chile, 2000-2010.

Tabla 2. Clasificación de diagnóstico de defunciones por hidatidosis según CIE10. Chile, 2000-2010

\begin{tabular}{cccccccc}
\multicolumn{7}{c}{ Clasificación de diagnóstico según CIE10 } & \\
Años & B670 & B671 & B673 & B676 & B678 & B679 & Total \\
2000 & 0 & 0 & 0 & 0 & 7 & 18 & 25 \\
2001 & 1 & 0 & 0 & 0 & 4 & 26 & 31 \\
2002 & 0 & 0 & 0 & 1 & 18 & 14 & 33 \\
2003 & 1 & 0 & 0 & 0 & 17 & 14 & 32 \\
2004 & 0 & 0 & 0 & 0 & 14 & 13 & 27 \\
2005 & 1 & 0 & 0 & 0 & 14 & 10 & 25 \\
2006 & 0 & 1 & 0 & 0 & 9 & 20 & 30 \\
2007 & 0 & 1 & 0 & 0 & 18 & 16 & 35 \\
2008 & 0 & 0 & 0 & 0 & 16 & 6 & 22 \\
2009 & 0 & 0 & 1 & 0 & 9 & 5 & 15 \\
2010 & 0 & 2 & 0 & 0 & 9 & 7 & 18 \\
Total & 3 & 4 & 1 & 1 & 135 & 149 & 293 \\
Porcentaje & 1,02 & 1,37 & 0,34 & 0,34 & 46,08 & 50,85 & 100,00 \\
\hline
\end{tabular}

\begin{tabular}{|c|c|c|c|c|c|c|}
\hline Edad & $\begin{array}{l}\text { n defunciones } \\
\text { país }\end{array}$ & $\begin{array}{l}\text { Tasa } \\
\text { país }\end{array}$ & $\begin{array}{c}\mathrm{n} \text { defunciones } \\
\text { Hombres }\end{array}$ & $\begin{array}{c}\text { Tasa } \\
\text { hombres }\end{array}$ & $\begin{array}{l}\text { n defunciones } \\
\text { Mujeres }\end{array}$ & $\begin{array}{c}\text { Tasa } \\
\text { Mujeres }\end{array}$ \\
\hline 0-9 años & 1 & 0,04 & 0 & 0 & 1 & 0,08 \\
\hline $10-19$ & 7 & 0,24 & 3 & 0,20 & 4 & 0,28 \\
\hline 20-29 & 14 & 0,56 & 4 & 0,32 & 10 & 0,81 \\
\hline $30-39$ & 19 & 0,77 & 15 & 1,21 & 4 & 0,32 \\
\hline $40-49$ & 26 & 1,11 & 17 & 1,46 & 9 & 0,76 \\
\hline 50-59 & 43 & 2,76 & 29 & 3,82 & 14 & 1,75 \\
\hline $60-69$ & 52 & 5,15 & 32 & 6,79 & 20 & 3,71 \\
\hline $70-79$ & 61 & 10,09 & 37 & 14,42 & 24 & 6,90 \\
\hline $80 y+$ & 70 & 27,70 & 27 & 30,04 & 43 & 26,41 \\
\hline Total & 293 & 1,80 & 164 & 2,04 & 129 & 1,57 \\
\hline
\end{tabular}

(0,22 muertes por 100 mil mujeres) (Tabla 1 y Figura 1$)$.

Del total de defunciones por hidatidosis, el mayor diagnóstico corresponde a la clasificación B67.9 -Equinococosis, otra y la no especificada- con $50,9 \%$, seguida por B67.8 -Equinococosis del hígado, no especificada- con $46,1 \%$ (Tabla 2).

La clasificación diagnóstica considerando el sexo muestra igual tendencia, es decir, el diagnóstico se concentra mayoritariamente en el código B67.9 (hombre $51,8 \%$; mujer $49,6 \%$ ) y B67.8 (hombre $45,1 \%$; mujer 47,3\%) (Datos no mostrados).

La edad mediana al fallecer en ambos sexos fue 68 años (rango 14-94 años), en hombres 66 (rango 17-91 años) y en mujeres 71 años (rango 14-94 años).

Según grupos de edad, la mayor tasa de mortalidad por grupo etario corresponde al grupo de 80 y más años, con 27,7 defunciones por 100 habts., a nivel país, 30 muertes por 100 mil hombres y 26,4 decesos por $100 \mathrm{mil}$ mujeres (Tabla 3).

Según la distribución regional sin considerar el sexo, las mayores tasas de mortalidad 2000-2010, corresponden a las regiones de La Araucanía (7,64 muertes por 100 mil habts.), Aysén (6,04 defunciones por 100 mil habts.), Los Lagos (3,83 muertes por 100 mil habts.) y Maule (3,72 decesos por 100 mil habts.), sobrepasando la tasa promedio del país ( 1,8 por 100 mil habts.).

En hombres, la distribución regional de las tasas de mortalidad cambia levemente, donde se mantiene la Región de La Araucanía con la mayor tasa de mortalidad (8,46 muertes por 100 mil hombres), seguido por las regiones del Maule (4,14 defunciones por $100 \mathrm{mil} \mathrm{hom-}$ bres), Aysén (3,82 decesos por 100 mil hombres) y Los Lagos (3,77 muertes por 100 mil hombres), todas sobre el promedio para el sexo en el período (2,04 defunciones por 100 mil hombres).

En las mujeres, la mayor concentración se registra en la Región de Aysén (8,51 defunciones por 100 mil mujeres), luego La Araucanía (6,84 muertes por 100 mil mujeres), Los Lagos (3,88 decesos por 100 mil mujeres) y Maule (3,30 defunciones por 100 mil mujeres), todas sobre el promedio para el sexo en el período (1,57 defunciones por 100 mil mujeres) (Tabla 4).

\section{Características socio-demográficas de los fallecidos}

Respecto al estado civil, hubo una leve concentración de defunciones en solteros $(48,5 \%)$. En hombres la categoría que concentró mayores defunciones es la correspondiente a los "casados" $(50,6 \%)$, mientras en las mujeres fallecidas predominó la categoría "soltera" $(51,2 \%)$.

De acuerdo al nivel de instrucción, del total de fallecidos por hidatidosis en el país, $59 \%$ tuvo un nivel de instrucción básico o primario, la segunda categoría en concentrar mayor número de defunciones fue la "ninguna" 
Tabla 4. Número de defunciones y tasas* de mortalidad por hidatidosis según región y sexo. Chile, 2000-2010

\begin{tabular}{|c|c|c|c|c|c|c|}
\hline Región & $\mathrm{n}$ defunciones país & Tasa país & n defunciones hombre & Tasa hombre & $\mathrm{n}$ defunciones mujer & Tasa mujer \\
\hline Tarapacá & 1 & 0,36 & 0 & 0,00 & 1 & 0,74 \\
\hline Antofagasta & 4 & 0,74 & 2 & 0,71 & 2 & 0,77 \\
\hline Atacama & 4 & 1,48 & 3 & 2,18 & 1 & 0,75 \\
\hline Coquimbo & 20 & 3,00 & 10 & 3,03 & 10 & 2,97 \\
\hline Valparaíso & 18 & 1,08 & 11 & 1,34 & 7 & 0,83 \\
\hline Libertador Bernardo O'Higgins & 15 & 1,78 & 10 & 2,36 & 5 & 1,20 \\
\hline Maule & 36 & 3,72 & 20 & 4,14 & 16 & 3,30 \\
\hline Bío Bío & 36 & 1,83 & 18 & 1,85 & 18 & 1,80 \\
\hline La Araucanía & 71 & 7,64 & 39 & 8,46 & 32 & 6,84 \\
\hline Los Lagos & 30 & 3,83 & 15 & 3,77 & 15 & 3,88 \\
\hline Aysén & 6 & 6,04 & 2 & 3,82 & 4 & 8,51 \\
\hline Magallanes & 4 & 2,56 & 2 & 2,44 & 2 & 2,70 \\
\hline Metropolitana & 42 & 0,64 & 28 & 0,88 & 14 & 0,42 \\
\hline Los Ríos & 6 & 1,61 & 4 & 2,15 & 2 & 1,08 \\
\hline País & 293 & 1,80 & 164 & 2,04 & 129 & 1,57 \\
\hline
\end{tabular}

(21,5\%). En los hombres, el nivel que concentró mayor número de defunciones también fue el "básico o primario" donde se incrementó a $62,2 \%$, continuado por el nivel "secundario" (14\%). En las mujeres fallecidas la categoría "básica o primaria" disminuyó a 55\%, seguido por el nivel "ninguna" (33\%). Ambos sexos fueron comparados respecto al nivel nacional.

Según el nivel de educación y la distribución regional, se observa que las regiones con mayores tasas de mortalidad registraron mayor proporción de fallecidos con nivel de instrucción "primario o básico" (Aysén 83,3\%; La Araucanía 56,3\%; Los Lagos 53,3\%).

Sobre el nivel de actividad, la mayoría al momento de la defunción se encontraba inactivo $(78,5 \%)$ y en mujeres esta proporción se incrementó $(97,7 \%)$ en relación a los hombres.

Al analizar el nivel de educación según su nivel de actividad en el total de muertes acumuladas, tanto para el nivel superior, secundario, básico y ninguna, se observa que la mayoría de los fallecidos se encontraba sin actividad laboral (100\%; 68; 80,4 y 93,7\%, respectivamente). Sólo el nivel medio registró una concentración levemente mayor de activos $(46,4 \%)$ versus los inactivos $(42,9 \%)$.

De acuerdo a la categoría ocupacional con actividad laboral, ésta se concentró mayoritariamente en el grupo de los obreros (47,3\%) (hombres 46,2\%; mujeres 66,7\%).

La ocupación en el grupo de inactivos, se concentró mayoritariamente en "jubilados" (74,3\%), de los cuales $25,1 \%$ fue aportado por la Región de La Araucanía. Si se observa la misma variable en el grupo de activos, se evidencia la mayor concentración en el sector de "inválido o recluido" $(50,9 \%)$ y, de este total, la Región de La Araucanía contribuyó con $25 \%$.

En igual análisis según el sexo, en hombres -la ocupación en inactivos-, se concentró mayoritariamente en "jubilados" (93,3\%), de los cuales $23,7 \%$ fue aportado por la Región de La Araucanía. Si se observa la misma variable en el grupo de activos, se evidencia la mayor concentración en el sector de "inválido o recluido" (51,9\%), y de este total la Región de La Araucanía contribuyó con $25,9 \%$.

En mujeres -la ocupación en inactivas-, se concentra mayoritariamente en jubiladas $(58,7 \%)$, de las cuales $27 \%$ fue aportado por la Región de La Araucanía. Al evaluar la misma variable en el grupo de activas, se evidencian concentraciones por igual $(33,3 \%)$ en el sector de "inválida o recluida" (100\% en la Región de Coquimbo) y "rentista" (100\% Región Metropolitana).

Según el análisis del área de residencia (urbano/rural), la mayoría de los fallecidos eran residentes urbanos $(56,1 \%)$. En hombres se mantuvo esta leve superioridad del sector urbano $(59,8 \%)$, mientras que en mujeres se estrecha esta relación (urbano 50,8\%; rural 49, 2\%).

$\mathrm{Al}$ evaluar el área de residencia por región, dentro del sector rural destacan con mayor proporción de defunciones las regiones de La Araucanía (41,5\%), Maule (20\%), Los Lagos (9,2\%), Coquimbo $(9,2 \%)$ y Bío Bío $(7,7 \%)$ (Figura 2). 


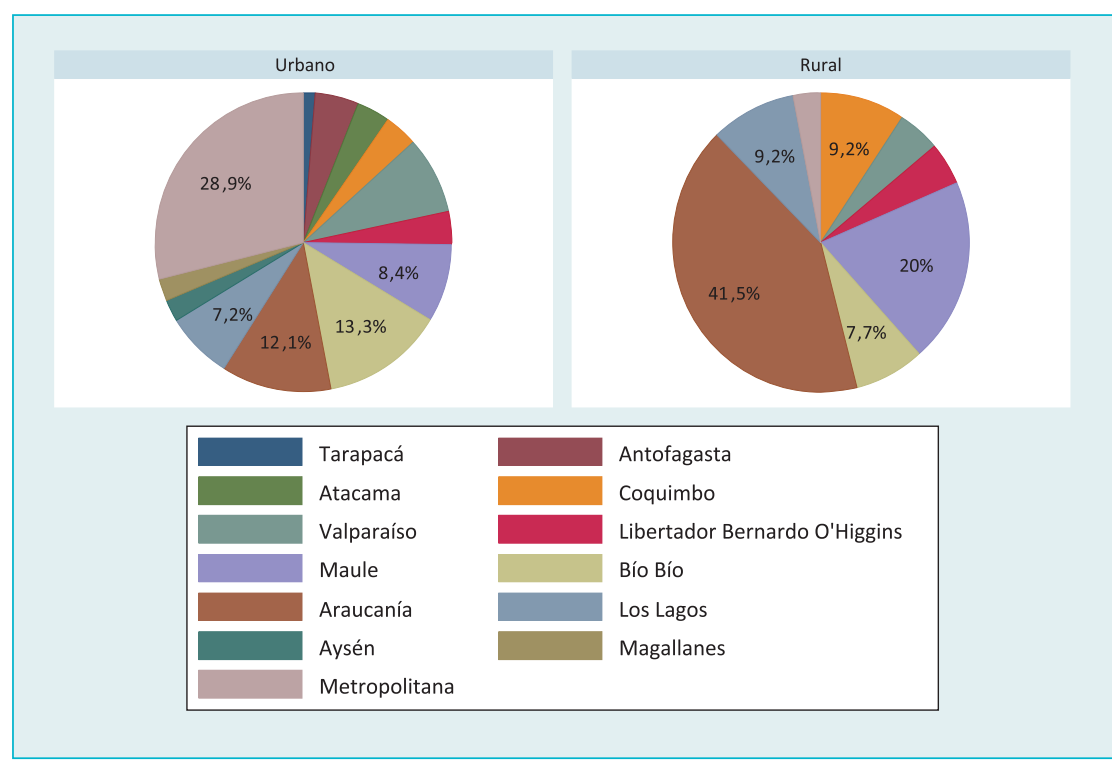

Figura 2. Distribución de muertes por hidatidosis según Área y Región. Chile, 2000-2010.
Tabla 5. Distribución de AVPP y razón de AVPP por hidatidosis según región. Chile, 2000-2010

\begin{tabular}{|lcc|}
\hline Región & AVPP & Razón AVPP \\
Tarapacá & $-2,62$ & $-0,009$ \\
Antofagasta & 12,12 & 0,022 \\
\hline Atacama & 6 & 0,022 \\
Coquimbo & 554,6 & 0,832 \\
Valparaíso & 304,46 & 0,183 \\
Libertador Bernardo O"Higgins & 188,5 & 0,224 \\
Maule & 307,88 & 0,318 \\
Bío Bío & 590,04 & 0,300 \\
La Araucanía & 958,69 & 1,032 \\
Los Lagos & 447,4 & 0,571 \\
Aysén & 77,84 & 0,784 \\
Magallanes & 93,64 & 0,600 \\
\hline Metropolitana & 725,2 & 0,111 \\
Los Ríos & 41,48 & 0,111 \\
\hline
\end{tabular}

Para la Región de La Araucanía del total de sus fallecidos $73 \%$ eran residentes de zonas rurales, mientras que para Coquimbo esta proporción corresponde a 66,7\%, Maule 65\% y para la Región de Los Lagos 50\%.

\section{Años de vida potencialmente perdidos-AVPP}

El impacto producido por muertes prematuras a consecuencia de esta zoonosis y por ende, pérdidas en años de contribución a la producción nacional es de 5.022,04 años de vida por el fallecimiento anticipado de 293 personas, lo cual expresado como una razón en relación a la población a mitad del período es de 0,31 años de pérdida por 1.000 habts.

Los AVPP han aumentado 5,5\% entre el 2000 (AVPP: 381,5) y 2010 (AVPP: 403,8). Los hombres (2734,02; razón AVPP 0,34 años de pérdida por 1.000 hombres) perdieron levemente más AVPP que las mujeres $(2642,42$; razón AVPP 0,32 años de pérdida por 1.000 mujeres).

Los grupos más afectados fueron: 50-59 años (21\%); 40-49 años (18,3\%) y 30-39 años (16,7\%). En hombres: $50-59$ años $(25,3 \%)$; 30-39 años $(22,9 \%)$; y $40-49$ años $(21,1 \%)$. En mujeres: $20-29$ años $(21,6 \%) ; 50-59$ años (17,4\%); y 40-49 años (12,9\%).

Esta estimación por región muestra que la mayor pérdida en años se registró en La Araucanía (AVPP 958,69), Región Metropolitana (AVPP 725,2), Bío Bío (AVPP 590,04) y Coquimbo (AVPP 554,6). Sin embargo, si se establece una jerarquización utilizando la razón de AVPP, se observa que las regiones que están sobre el nivel país, en orden descendente son: La Araucanía (razón 1,03 años de pérdida por 1.000 habts), Coquimbo (razón 0,8 años de pérdida por 1.000 habts), Aysén (razón de 0,78 años de pérdida por 1.000 habts), Magallanes (razón 0,6 años de pérdida por 1.000 habts), Los Lagos (razón 0,57 años de pérdida por 1.000 habts) y Maule (razón 0,32 años de pérdida por 1.000 habts) (Tabla 5).

\section{Discusión}

Las enfermedades que afectan a los animales y especialmente, las que son trasmitidas al hombre, representan un freno para el desarrollo social y económico de sus habitantes, provocando disminución en la producción animal, ecosistemas, economía, turismo y reducción del comercio, en conjunto con la puesta en riesgo de la seguridad alimentaria de la población de escasos recursos. Además, generan pérdidas por un bajo rendimiento laboral, morbilidad, impacto negativo en la calidad de vida y en ocasiones defunciones humanas.

Esta antropozoonosis es la enfermedad parasitaria de mayor importancia en el país, la cual puede ser absolutamente prevenida. En Chile, la hidatidosis es un afección hiperendémica que, a pesar de su connotación social y económica, sigue siendo un problema de salud pública no abordado en forma integral y que, en consecuencia, permanece no resuelto.

Su distribución geográfica no es similar en todo el país, hay un aumento de la incidencia a medida que se avanza hacia el sur. La distribución está asociada a la población ganadera, donde las regiones de Aysén y Magallanes concentran más de la mitad de la ganadería ovina del país 
asociados a sistemas de explotación tradicionales y extensivos de manejo con una importante población canina.

Los casos notificados de hidatidosis humana han variado desde 297 en el año 2000 (51,5\% hombres) a 240 en el 2010 (53,8\% hombres), con un promedio anual de 304 casos. Los egresos hospitalarios han variado de 1.150 en 2001 (53,2\% hombres) a 799 en 2010 (53,9\% hombres), con un promedio anual cercano a 1.003 egresos. Mientras, las muertes clasificadas según CIE10 (códigos B67.0 a B67.9) han variado de 25 en el año 2000 ( $52 \%$ hombres) a 18 en el 2010 (55,6\% hombres), con un promedio anual cercano a 26,6 defunciones.

Los indicadores anteriores estiman una tendencia al decrecimiento; sin embargo, esconden las realidades regionales, donde no sólo existe una distribución geográfica heterogénea asociada a la economía básica, evidenciada por el aumento de los casos a medida que se avanza hacia el sur, si no también, por las diferencias en la calidad y acceso a las prestaciones de salud. Ejemplo de esta disparidad es la tasa de mortalidad acumulada 2000-2010 en la Región de La Araucanía (7,6 defunciones por 100 mil habts) en comparación con la del país $(1,8$ defunciones por 100 mil habts).

La Región de Aysén tiene la mayor tasa de notificación ENO y egresos hospitalarios; no obstante, no es la región que presenta la mayor tasa de mortalidad, lo cual puede significar entre otras razones que disponen de servicios de salud adecuados a la realidad epidemiológica local incluyendo un sistema de pesquisa más eficiente y mayor acceso al sistema sanitario. Situación totalmente diferente a la Región de La Araucanía, la cual tiene la sexta posición en términos de la tasa de notificación de casos ENO 2000$2009^{13}$ y sin embargo, para el período evaluado registra la mayor tasa de mortalidad del total de las regiones.

La mayoría de los pacientes tiene un solo órgano afectado y ubicado en hígado o pulmones ${ }^{1}$. En general, en los estados incipientes de la enfermedad no se registran síntomas y los quistes, que crecen a razón de 1-30 $\mathrm{mm} / \mathrm{año}$, permanecen pequeños y asintomáticos durante muchos años. Producto de esta tasa de crecimiento lenta, la mayoría de los casos son diagnosticados en la adultez ${ }^{1}$, situación confirmada en Chile por la edad mediana de notificación de casos ENO de 38 años y de egresos hospitalarios de 40 años ${ }^{13}$. Sin embargo, en nuestro país se continúan diagnosticando casos en menores de edad; de hecho, el sistema ENO 2010 notificó 26,3\% de casos en personas bajo 19 años de edad, lo cual habla de infección reciente. Adicionalmente, en las defunciones acumuladas para el período estudiado se registra muertes en $2,7 \%$ de pacientes bajo 19 años.

El diagnóstico se basa en datos epidemiológicos, clínicos, radiológicos y de laboratorio ${ }^{6}$. Se ha determinado que la ecografía es una técnica muy importante para el diagnóstico y seguimiento post tratamiento, la cual podría ser eventualmente apoyada por resonancia magnética ${ }^{19}$. Por lo tanto, las técnicas mencionadas hablan de la necesidad de cierto nivel técnico -fundamentalmente de imagenología-, el cual no necesariamente está disponible en todos los Servicios de Salud. De hecho, el informe del MINSAL sobre brechas de especialidades médicas hace referencia a la falta de 3.745 h en radiología. En el Servicio de Salud de La Araucanía, de acuerdo a datos del MINSAL se dispone de 14 profesionales en imagenología, lo cual representa un 5,8\% del total de estos especialistas disponibles a nivel país en los Servicios de Salud (País $\left.\mathrm{N}^{\circ} 240\right)^{20}$.

El tratamiento de esta enfermedad es generalmente quirúrgico, con un alto costo tanto para el paciente, como para el sistema de salud. Si relacionamos la necesidad de resolución probablemente quirúrgica de los fallecidos en relación a la disponibilidad de médicos especialistas, se observa que en todo el país existe una brecha de $6.072 \mathrm{~h}$ de cirugía general. En la Región de La Araucanía existen 60 profesionales de cirugía general y subespecialidades. A nivel país se dispone de 1.455 profesionales para igual especialidad; por lo tanto, en esta región se dispone de $4,1 \%$ del total de esta especialidad en relación al total nacional disponible en los Servicios de Salud ${ }^{20}$.

En el Servicio de Salud de La Araucanía existe un elevado número de pacientes en lista de espera quirúrgica, lo cual puede responder a diferentes razones, entre ellas: déficit de especialistas; reforma de la salud que posterga algunas patologías en prioridad de la resolución de las enfermedades GES; disminución del recurso para la atención de consulta de nuevos pacientes; falta de reposición y mantención de equipamiento de alta especialidad, especialmente el área quirúrgica; en comunas con $<20.000$ habts., y habitualmente con alto porcentaje de población rural, los profesionales médicos deben comúnmente realizar atención en las postas de salud rural, y en consecuencia, el número de médicos que permanece en el establecimiento es insuficiente para resolver la demanda espontánea de salud ${ }^{21}$. De hecho, la Atención Primaria de Salud (APS) en La Araucanía dispone de 160 médicos, lo cual representa $4,8 \%$ del total de médicos de la APS nacional $\left(\mathrm{N}^{\circ}\right.$ Chile 3.335). El 5,7\% de la población chilena reside en esta región. Adicionalmente y según datos del MINSAL, los Servicios de Salud de La Araucanía están bajo el promedio país de jornadas de $44 \mathrm{~h}$ de médicos por cada 10.000 personas (Chile 2,6; La Araucanía Sur 2,5 y La Araucanía Norte 1 jornada de 44 h/10.000 personas) ${ }^{20}$. Estando en general la región bajo el promedio nacional de horas médicas $2010^{20}$.

McManus y cols., señalan que si el manejo médico no es el adecuado, la enfermedad se puede transformar en una condición grave, con mal pronóstico y elevada mortalidad $^{19}$. 
El concepto de "muerte evitable" se ha discutido por décadas y hace referencia a una atención médica efectiva y oportuna, que potencialmente evita muertes prematuras que no deben ocurrir. Con el conocimiento y la tecnología sanitaria disponible, una proporción importante -sino el total- de muertes por hidatidosis podrían ser evitadas. Internacionalmente existe estandarización mediante listados de enfermedades cuya muerte es evitable, en la cual está incluida la hidatidosis. La definición de estas enfermedades se basa en las intervenciones preventivas y curativas, por lo cual, una muerte por hidatidosis es evitable dado que, al menos en teoría, se dispone de capacidad resolutiva en términos de diagnóstico y tratamiento. A la vez, una muerte por complicación de la enfermedad, también es evitable dada la existencia de adelantos quirúrgicos, radiológicos, inmunológicos y farmacológicos ${ }^{15}$, entre otros.

La hidatidosis es una enfermedad multifactorial en términos de su presentación y propagación destacando la dinámica de las relaciones con distintas especies animales; el sistema sanitario (acceso, calidad y equidad); cambios demográficos con incremento de la población humana en zonas urbanas y periurbanas; el nivel socio-económico y cultural; el movimiento nacional e internacional de personas, animales, alimentos y mercancías, además del efecto del cambio climático. Por lo anterior, esta enfermedad representa una mayor carga para las personas que viven en situación de pobreza. Esto último explica fácilmente por qué la Región de La Araucanía posee la mayor tasa de mortalidad en el país, a pesar que no es la que registra mayor número de casos notificados ni de egresos hospitalarios. Esta región, de acuerdo al Plan Araucanía del Gobierno está en una situación de atraso de décadas respecto al país. De hecho, a nivel del PIB e ingreso per cápita (p/c) se evidencia un retraso respecto al país de 20 años, lo cual significa que desde 1990 La Araucanía presenta el PIB $\mathrm{p} / \mathrm{c}$ más bajo a nivel nacional y durante este período se han registrado como promedio 2,1 millones de pesos por debajo del nivel del país. En relación al atraso de la extrema pobreza (tasa país 3,7\%'; La Araucanía 9\%; Mapuches Araucanía 10,3\%; Mapuches país 5,9\%) y pobreza (tasa país 15,1\%; La Araucanía 27,1\%; Mapuches Araucanía $30 \%$; Mapuches país 20,5\%), se evidencian 18 y 16 años respectivamente, mientras en años de escolaridad 16 años de retraso y en términos de desigualdad educacional más de 20 años $^{21}$. La Región de La Araucanía registra el nivel más bajo de ingresos monetarios p/c en Chile, situación que persiste y se estabiliza desde la década de los '90. El Gobierno establece una relación estrecha entre el nivel de ingreso regionales y los altos niveles de pobreza e indigencia. Sin embargo, el nivel de ingresos no sólo está relacionado con la pobreza, sino que de acuerdo al Programa Eurosocial son los ingresos la variable que más influye en la distribución de la mortalidad ${ }^{21,22}$.

En nuestro análisis de mortalidad los afectados ma- yoritariamente poseen bajo nivel de educación, con 59\% de instrucción primaria, lo cual es mayor en hombres y en las regiones de Aysén (83,3\%) y La Araucanía $(56,3 \%)$. En esta última región no sorprenden estos datos, dado que los distintos indicadores educaciones regionales la muestran como extremadamente atrasada respecto del país, situación que se agudiza en la población mapuche ${ }^{21}$.

Si bien las defunciones se registran mayoritariamente en población urbana a lo largo del país, esta distribución cambia fundamentalmente en las regiones de La Araucanía, donde del total de sus fallecidos $73 \%$ corresponde a residentes de zonas rurales, mientras que para Coquimbo esta proporción corresponde a $66,7 \%$ y Maule $65 \%$. Dadas las características geográficas y culturales de la población rural, se puede dificultar el acceso oportuno a los servicios de salud y contribuir a una mayor mortalidad.

Adicionalmente, se reafirma la tesis que la Región de La Araucanía es la que posee mayores muertes evitables, es decir, injustas y por falta de acceso oportuno y de calidad a la atención de salud, mediante la razón de AVPP, la cual la sitúa en primer lugar nacional y $232 \%$ sobre la razón AVPP Chile.

La información de mortalidad es importante para entender de mejor manera la carga de enfermedad y evaluar la efectividad de las intervenciones de salud pública. Consecuentemente, a través de las tasas de mortalidad se puede evaluar la efectividad de las acciones en salud, en términos de acceso, calidad y oportunidad.

Finalmente, es deber del Estado proteger la salud y el bienestar de sus habitantes.

\section{Resumen}

Introducción: La hidatidosis es una zoonosis universal de alto impacto socio-económico en Chile y en otras latitudes, siendo hoy para nuestro país una afección de notificación obligatoria en humanos. Objetivo: Caracterizar las defunciones por hidatidosis y establecer si existen diferencias de acuerdo a la región de residencia. Material y Métodos: Se utilizaron datos del Sistema de Defunciones (2000-2010) realizándose un análisis descriptivo. Resultados: El total de defunciones es de 293 (56\% hombres). La tasa de mortalidad se ha desplazado desde 0,16 en 2000 a 0,11 en 2010 muertes por 100 mil habitantes. El mayor diagnóstico fue B67.9 (Equinococosis, otra y la no especificada). El nivel de instrucción de los afectados fue mayoritariamente básico. La Región de La Araucanía registra la mayor tasa de mortalidad. Se produjo la pérdida de 5.022,04 años de vida por el fallecimiento prematuro de 293 personas, con una razón de AVPP de 0,31 años de pérdida por 1.000 habitantes. En la Región de La Araucanía esta razón de AVPP se incrementa a 1,03 años 
de pérdida por 1.000 habitantes. Conclusiones: La tasa de mortalidad tiende a disminuir en el período estudiado; sin embargo, oculta diferencias regionales. Se destaca que las muertes por hidatidosis son muertes evitables y por lo tanto injustas. Finalmente, el análisis de mortalidad es importante para comprender de mejor forma la carga de la enfermedad y evaluar la efectividad de las intervenciones de salud pública.

\section{Referencias bibliográficas}

1.- Menezes da Silva A. Human echinococcosis: A neglected disease. Gastroenterol Res Pract 2010; 2010: 583297.

2.- Osorio M, Godoy H. Estudio "Vulnerabilidad Social Frente a Hidatidosis Humana". SEREMI de Salud. Región de Aysén. Informe Final Versión 2.0, Marzo 2008. <seremiaysen. redsalud.gob.cl/.../9dada14d3923c7d...> [Consultado: 25 enero de 2013].

3.- Cortés S, Valle C. Hidatidosis humana: Generalidades y situación epidemiológica en Chile según egresos hospitalarios y notificación obligatoria entre los años 2001 y 2005. Rev Chilena Infectol 2010; 27 (4): 329-35.

4.- Sánchez C. Hidatidosis. Pequeños rumiantes 2002; 3 (2): 9-15.

5.- Rodríguez J. Hidatidosis humana: Enfermedad parasitaria se ha transformado en un serio problema de salud pública. El Rancagüino Online. 9 noviembre de $2009<$ http:// www.elrancaguino.cl/news/Hidatidosishumanaenfermedad-parasitaria-se-hatransformado-en-un-serio-problema-de-saludpublica/> [Consultado: 14 octubre de 2012]

6.- Organización Panamericana de la Salud. Organización Mundial de la Salud. Informe del Proyecto Subregional Cono Sur de Control y Vigilancia de la Hidatidosis Argentina, Brasil, Chile y Uruguay. Primera Reunión Constitutiva. Montevideo, Uruguay, 7 al 9 de julio de 2004.

7.- Amez J, Castañeda E. Tratamiento quirúrgico de la hidatidosis pulmonar en el Hospital Nacional Cayetano Heredia 1989-1999. Revista Médica Heredia 2002; 13 (1): 3-9.

8.- Acuña M, Briceño C, Domínguez M, Montoya L. Hidatidosis uterina: una localización excepcional. Rev Chilena Obstet Ginecol 2008; 73 (6): 389-92.

9.- Gobierno de Chile. Ministerio de Salud. Reglamento sobre notificación de enfermedades transmisibles de declaración obligatoria $\mathrm{N}^{\circ} 158$. Santiago, 22 de octubre de 2004.

10.- Gobierno de Chile. Ministerio de Salud. Normas Técnicas de Vigilancia de Enfermedades Transmisibles. 2000. <http://epi.minsal.cl/epi/ html/public/enftransmisibles.pdf $>$ [consultado: 19 enero de 2013].

11.- Irabedra P, Salvatella R. El proyecto subregional cono sur de control y vigilancia de la hidatidosis. Revista Peruana Medicina Experimental Salud Pública. 2010; 27 (4): 598-603.

12.- Berns D, Rager B. Emerging infectious diseases: a cause for concern. Israel Med Assoc J 2000; 2: 919-23.

13.- Martínez P. Hidatidosis humana: antecedentes generales y situación epidemiológica en Chile, 2001-2009. Rev Chilena Infectol 2011; 28 (6) 585-91.

14.- Roses M. Organización Panamericana de la Salud. Las enfermedades desatendidas en las poblaciones postergadas, con énfasis en las zoonosis. Informe final y documentos seleccionados. $14^{\text {a }}$ Reunión Interamericana a Nivel Ministerial en Salud y Agricultura (RIMSA 14) Agricultura y Salud: Sinergia para Desarrollo Local Ciudad de México, D.F., México, 21-22 de abril de 2005. 163-74 pp.

15.- Gobierno de México. Secretaría de Salud. Subsecretaría de Innovación y Calidad. Dirección General de Información en Salud. "La Mortalidad en México, 2000-2004. "Muertes evitables: magnitud, distribución y tendencias". México, 2006. 368 pp.
16.- Whitehead M. World Health Organization. The concepts and principles of equity and health. Document EUR/ICP/RPD/414. Copenhagen, 1990.

17.- Organización Panamericana de la Salud (OPS). Clasificación Estadística Internacional de Enfermedades y Problemas relacionados con la Salud. Décima revisión. 1995.

18.- Gobierno de Chile. Instituto Nacional de Estadística (INE). Departamento de Demografía. Programa de proyecciones de la población. Proyecciones de población por grupos de edad y sexo. <http://www.ine.cl/ canales/chile_estadistico/demografia_y_vitales/ proyecciones/MenPrincOK.xls> [Consultado: 20 diciembre de 2012].

19.- McManus D, Gray D, Zhang W, Yang Y. Diagnosis, treatment, and management of echinococcosis. Br Med J 2012; 344:e3866.

20.- Gobierno de Chile. Ministerio de Salud. Subsecretaría de Redes Asistenciales. Dr. Luis Castillo F. "Déficit crónico de especialistas. Visión desde el MINSAL". 2012. <www.asemech.cl/.../Deficit-cronicode-especialistas... $>$ [Consultado: 25 enero de 2013].

21.- Gobierno de Chile. Ministerio Secretaria General de la Presidencia. Plan de La Araucanía. nd. <www.minsegpres.gob.cl/wp.../ Plan_Araucania.pdf $>$ [Consultado: 25 enero de 2013].

22.- Garay J. Comisión Europea. Europaid. Salud Global, en la UE y en LA. Objetivos y dinámicas. Situación, tendencia y desafíos comunes. Taller de arranque del programa Eurosocial II-Salud Equidad en Salud: medición para la acción Montevideo, 19-21 de noviembre, 2012. 